University of South Carolina

Scholar Commons

1985

\title{
Modeling and System Identification of Residential Air Conditioning Load
}

Anil Pahwa

Kansas State University

Charles W. Brice

University of South Carolina - Columbia, brice@engr.sc.edu

Follow this and additional works at: https://scholarcommons.sc.edu/elct_facpub

Part of the Electrical and Computer Engineering Commons

\section{Publication Info}

Published in IEEE Transactions on Power Apparatus and Systems, Volume 104, 1985, pages 1418-1425. http://ieeexplore.ieee.org/xpl/Recentlssue.jsp?punumber=10669

(c) 1985 by IEEE

This Article is brought to you by the Electrical Engineering, Department of at Scholar Commons. It has been accepted for inclusion in Faculty Publications by an authorized administrator of Scholar Commons. For more information, please contact digres@mailbox.sc.edu. 
At each new time step, the iterative process for the new ac-dc solution begins with the dc solver using the ac voltages of the previous time step as initial estimates to determine the corresponding ac currents injected by the converters into the ac network. Based on the new estimates of the converter ac currents, the ac transient stability program calculates a new set of ac network voltages. The new set of ac voltages at the converter buses is then used by the dc system solver to determine fresh estimates of the injected currents of the converters. This iterative process between the ac and dc networks solvers is repeated until the ac voltages converge within some preset tolerance.

Numerical examples based on a 3-terminal, 3-machine acdc system were used to demonstrate the capability of an acdc transient stability program using the proposed algorithm. These examples showed the successful handling of mode shifts between various kinds of control characteristics, restart after a severe fault, and also power modulation to damp the electromechanical oscillations of ac generators.

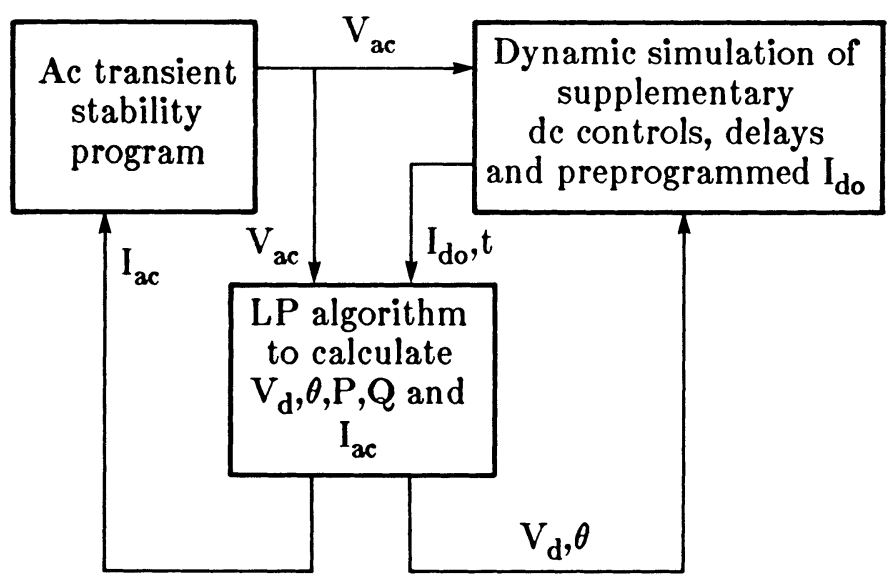

Fig. A Ac-dc transient stability program

$84 S M 591-4$

June 1985, pp. 1418-1425

\section{Modeling and System Identification of Residential Air Conditioning Load}

Anil Pahwa, Member, IEEE

Kansas State University, Manhattan, KS

C. W. Brice, III, Senior Member, IEEE

University of South Carolina, Columbia, SC

(Formerly with Dept. of Electrical Eng.,

Texas A\&M University)

In this paper a fairly detailed physically-based model of the residential air conditioning system is presented. The dynamic behavior of the air conditioned house has been modeled from fundamental physical principles, i.e., the thermodynamics of the house and the effect of various environmental variables which affect transfer of heat into and out of the house. The effect of temperature, solar radiation, air infiltration, humidity and internal heat is taken into account while developing the model. The effect of other minor variables is incorporated by introducing noise terms in the model equations. On mathematically representing the effect of above mentioned variables, a lumped-parameter equivalent circuit model is obtained, which is shown in Fig. 1.
Engineering judgement and resulting approximations are used to obtain expressions for rate of switching on $(\lambda(k))$ and rate of switching off $(\mu(k))$ of the air conditioner at hour $k$ as a function of other variables and model parameters. The equations thus obtained represent a state variable model given by

$$
\begin{aligned}
T w(k+1)= & \exp (-2 / R w C w) T w(k) \\
& +\frac{(1-\exp (-2 / R w C w))}{2} \\
& \cdot[T o(k)+T s+R w(/ r(k)+\operatorname{Im}(k))] \\
\lambda(k)= & \frac{1}{2 R w C i E b} T w(k)+\frac{1}{2 R c C i E b} T o(k) \\
& -\left(\frac{1}{2 R w C i E b}+\frac{1}{2 R c C i E b}\right) T s \\
& +\frac{1}{2 C i E b} \text { linst }(k)-\frac{1}{2 R w C i}-\frac{1}{2 R c C i}
\end{aligned}
$$

and

$$
\begin{aligned}
\mu(k)= & -\frac{1}{2 R w C i E b} T w(k)-\frac{1}{2 R c C i E b} T o(k) \\
& +\left(\frac{1}{2 R w C i E b}+\frac{1}{2 R c C i E b}\right) T s-\frac{1}{2 C i E b} \text { linst }(k) \\
& +\frac{1}{2 C i E b}(\operatorname{lac}(k)-\text { Icond }(k))-\frac{1}{2 R w C i}-\frac{1}{2 R c C i}
\end{aligned}
$$

The parameters in the model represent physical quantities but their measurement would be difficult. Therefore, system identification is used to estimate the values of the parameters. A computer program based on the maximum Likelihood indentification has been developed for this purpose. Simulation has been used to obtain data. However, the algorithm has been tested with different model parameters and simulation conditions. Then, the output of the indentified model has been compared with the simulated data to check the accuracy of the identified model. It has been found that the two outputs match quite closely. In fact, the latter has been found to be filtered version of the former in all the cases.

\section{Aggregation}

The aggregate demand of a group of identical air conditioners is determined using duty factor, which is the percentage of time the air conditioner stays on in a cycle of the air conditioner. In terms of the output of the model the duty factor at hour $k$ is

$$
D F(k)=\lambda(k) /(\lambda(k)+\mu(k))
$$

Now, the aggregate demand is determined by multiplying mean value of the duty factor with the total installed capacity of the air conditioners. In this paper a group of houses is chosen and sample mean and sample variance of the duty factor are determined for the simulated data and the data obtained using the identified. It has been found that both match quite closely.

\section{Energy Consumption Vs Parameters}

The model is then used to study the energy consumption of the air conditioners, which is also computed using the duty factors. A test case is chosen and the parameters are varied one at a time. The energy consumption for all the cases is determined and the correlation between change in the parameter values and energy consumption is studied. 


\section{Voltage Response}

Next, the effect of voltage variation on the performance of the air conditioners is determined. A voltage dependent model of single-phase induction motor is interfaced with the air condtioner model and the outputs are determined at $10 \%$ reduction in voltage. It has been found that steady state load of the air conditioners is insensitive to voltage reduction.

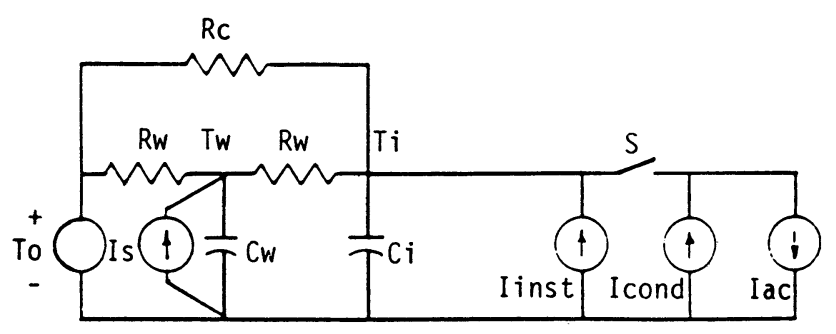

Fig. 1 Electrical Circuit Model of a House

\title{
The Interaction of Torsional Resonances With Network Resonances in Synchronous Generators
}

\author{
Boon-Teck Ooi \\ McGill University, Montreal, P.Q., Canada \\ Geza Joos \\ Ecole de Technologie Superieure, \\ Montreal, P.Q., Canada
}

The shaft failures in Southern California's Mohave plant alerted the power utility community to the dangers of instability from the induction generation effect and the torsional interaction effect. The failures also pointed to the critical situation of a mechanical resonant frequency $\omega_{\nu}$ being able to stimulate an electrical network resonant frequency, $\omega_{e}$ $=\omega_{0}+\omega_{\nu}$ through the upper sideband (along line segment $A D E$ in Fig. 1) or through the lower sideband, $\omega_{e}=\left|\omega_{0}-\omega_{\nu}\right|$ (along line segments $A B$ and $B C$ ).

Because the induction generation effect is the better understood of the two, one has a tendency to shelter under the immunity provided by induction machine theory. One forgets that the question of protection against the torsional interaction effect has not been addressed. An example of this is the advocacy of shunt capacitor compensation, on the basis that since the network resonance frequency is supersynchronous, the amortisseur windings behave as an induction machine and the resonant mode has to be positively damped. At least one utility has proceeded in using shunt capacitor compensation with the comforting assurance that the lowest network frequency is $90 \mathrm{~Hz}$. Unfortunately, there remains unanswered the question of its safety with respect to torsional resonance frequencies at $90-60=30 \mathrm{~Hz}$ and to $90+60=150 \mathrm{~Hz}$.

Hitherto, research on torsional interaction has been limited to the subsynchronous region of operation, along the line segment $A B$ in Fig. 1, where it is notoriously unstable. Apart from the prejudices based erroneously on induction machine theory, there is no reason to believe that the torsional interaction, along the line segments $A D E$ and $B C$ is positively damped. The purpose of this paper is to extend the research to these regions. This is because it is beginning to be recognized that the electrical network is spiked with a multiplicity of supersynchronous resonances which come from the distributed capacitances of the transmission lines. Because of space limitation, this paper is restricted to interactions along $\mathrm{BC}$. A future publication will treat line segment ADE.

The research tool used in this paper is the phase modulation theory of the torsional interaction phenomenon [1]. Part I of the paper establishes a high level of confidence in the theory by showing close agreement of its predictions with those calculated by a radically different method. Part II develops the conclusion that supersynchronous torsional interaction is positively damped, except for the Cross Terms Limitation.

This is done by showing that, for all supersynchronous torsional frequencies, the total damping torque is:

$$
\sum_{i=1}^{8} T_{i}=T_{\text {direct }}+T_{\text {cross }}
$$

where

$$
\begin{gathered}
T_{\text {direct }}=\left[a\left(\omega_{\nu}\right) /_{f}\right]^{2}+\left[b\left(\omega_{\nu}\right) /_{d}\right]^{2}+\left[b\left(\omega_{\nu}\right) /_{q}\right]^{2} \\
T_{\text {cross }}=2 c\left(\omega_{\nu}\right) I_{f} I_{d}
\end{gathered}
$$

and $a\left(\omega_{\nu}\right), b\left(\omega_{\nu}\right)$ and $c\left(\omega_{\nu}\right)$ are real numbers based on the parameters of the generator and the system.

Since eq. (2) is always positive and contains the squares of the field current $I_{f}$ and the armature currents $I_{d}, I_{g}$, it plays a dominant part in making eq. (1) positive. The Cross Terms Limitation comes from eq. (3) which has always a negative region and therefore a chance of making eq. (1) negative. However eq. (3) is a linear function of $I_{f}$ and $I_{d}$ so that ultimately the square terms in eq. (2) prevail, thus making positive damping to be the rule rather than the exception.

This is illustrated in Fig. 2 which plots $T_{\text {direct }}$ and $-T_{\text {cross }}$ as a function of $I_{d}$, keeping $I_{f}$ and $I_{g}$ constant. In practice, $I_{f} \approx 1.0$ pu with the result that $T_{\text {direct }}$ is a parabola displaced upwards in the ordinate. The curve (i) shows the case where $-T_{\text {cross }}$ never intersects $T_{\text {direct }}$. Curves (ii) and (iii) are for cases where it cuts $T_{\text {direct }}$ at two points, making the region between the two points, unstable, One needs detailed knowledge of the system and machine parameters to determine which is the appropriate situation. In the absence of this knowledge, one can nevertheless say with certainty that:

(a) The small region around the origin of the $\left(I_{f}, I_{d}, I_{g}\right)$-space is always positively damped with respect to supersynchronous torsional resonance interactions.

(b) When

$$
\left[a\left(\omega_{\nu}\right) b\left(\omega_{\nu}\right)\right]^{2}>\left[c\left(\omega_{\nu}\right)\right]^{2}
$$

the positively damped region is of infinite extent.

(c) When the inequality of eq. (4) is reversed, the region containing the origin and bounded by:

$$
I_{d}<\frac{a^{2}\left(\omega_{\nu}\right) I_{f}}{\left|c\left(\omega_{\nu}\right)\right|+\mid \sqrt{c^{2}\left(\omega_{\nu}\right)-a^{2}\left(\omega_{\nu}\right) b^{2}\left(\omega_{\nu}\right)}}
$$

is positively damped.

\section{Reference}

[1] B. T. Ooi, "Phase modulation theory of electromechanical damping in synchronous generation," IEEE Trans. Power Apparatus and Systems, Vol. PAS-100, No. 5, May 1981, pp. 2211-2218. 